\title{
Timing Means Everything, Maybe
}

\author{
Steven R. Alberts, MD, MPH \\ Division of Medical Oncology, Mayo Clinic, Rochester, MN
}

Many patients with colorectal cancer will have liver metastases at the time of their initial diagnosis (synchronous) or will develop liver metastases at some point after undergoing potentially curative therapy for their primary disease (metachronous). For a large proportion of patients with metastatic colorectal cancer, whether synchronous or metachronous, the metastases will be restricted to the liver. A number of questions exist on how best to care for patients with liver metastases from colorectal cancer. These include the role and timing of chemotherapy, as well as the role and timing of surgery. To date, much of what we have learned about the appropriate care of patients with liver metastases has come from retrospective summaries of institutional experience. However, a small but growing number of clinical trials exist.

One area of uncertainty surrounds the management of patients with synchronous liver metastases that may be resectable. In particular, is it best to initially resect both the primary and the metastases, to initially give chemotherapy, or a combination of these two approaches such as to resect the liver metastases, give chemotherapy, and then remove the primary $?^{1}$ In general, patients with synchronous disease do not do as well as patients with metachronous disease. ${ }^{2}$ Overall survival appears to be shorter and the rate of recurrence after a potentially curative resection appears to be higher. It has therefore been advocated by some to use neoadjuvant chemotherapy as a surrogate method of determining outcome. In prior retrospective series, patients with stable or responding disease have done better than those with progressive disease. Based on this information it had been suggested that the appropriate approach is to give neoadjuvant chemotherapy to assess response.

(C) Society of Surgical Oncology 2009

Published Online: 23 June 2009

S. R. Alberts, MD, MPH

e-mail: Alberts.steven@mayo.edu
In the current issue of Annals of Surgical Oncology Dr. Gallagher and colleagues summarize the approximately 9year experience of management of patients with potentially resectable synchronous liver metastases from colorectal cancer. ${ }^{3}$ The retrospective analysis specifically addresses the relationship of response to preoperative chemotherapy to outcome in 111 patients undergoing resection. In this series the response of patients receiving neoadjuvant chemotherapy did not predict overall survival. Patients with a partial or complete response had a median overall survival of 58 months compared with 61 months for patients with evidence of progression. This result is surprising for several reasons. First, it appears to contradict prior reports. Second, the patients in this series undergoing resection with evidence of progressive disease had a higher rate of positive surgical margins (39\%) compared with those with responding disease $(5 \%)$ and yet seemed to have lived as long. We are therefore let to ponder how long-held beliefs of factors predicting outcome could now be less relevant.

In an earlier retrospective series Dr. Adam and colleagues showed that response to neoadjuvant therapy is a meaningful predictor of outcome in patients with potentially resectable liver metastases from either synchronous or metachronous disease. ${ }^{4}$ In this series $78 \%$ of patients with stable or responding disease had synchronous disease compared with $53 \%$ of patients with progressive disease. In addition, the proportion of patients with a curative resection was lower in patients with progressive disease.

In a separate retrospective study Dr. Allen and colleagues specifically reviewed the impact of neoadjuvant chemotherapy in patients with synchronous disease. ${ }^{2}$ Using a group of 106 patients, 52 who received neoadjuvant chemotherapy and 54 who went initially to surgery, 5-year survival was shown to be statistically similar between the two groups. Of note $28 \%$ of patients going directly to surgery did not receive postoperative chemotherapy. However, when a comparison was made between patients receiving neoadjuvant chemotherapy who did not progress 
and those not receiving neoadjuvant chemotherapy, a significant benefit to neoadjuvant chemotherapy was observed. Of note, 46 of the 52 patients receiving neoadjuvant chemotherapy were subsequently included in the current report. The question then becomes: What is the appropriate comparison to determine the potential benefit of neoadjuvant chemotherapy? In this situation, when the question is specifically "Does neoadjuvant therapy impact outcome as measured by overall survival?" the analysis by Dr. Gallagher appears to be the more appropriate approach.

It is also important to point out that in the study by Dr. Gallagher 91 of the 111 patients $(82 \%)$ received chemotherapy. This included the use of both systemic chemotherapy and chemotherapy given by hepatic artery infusion. Therefore it may be more appropriate to restate the analysis as an assessment of perioperative therapy on overall survival. It is unclear in the report from Dr. Adam what proportion of patients received postoperative chemotherapy.

There are several other noteworthy differences between the studies of Drs. Gallagher and Adam. Dr. Adam's study included patients with a higher number of metastases on average and a higher rate of potentially curative resections (94\% versus $85 \%$ ). This difference should have more strongly influenced Dr. Gallagher's study to show a difference in outcome. The use of postoperative chemotherapy may have influenced the outcome by eradicating residual disease at a positive surgical margin. However, it remains uncertain from the report what approach was taken in patients with progressive disease. Only a brief summary is provided for the nine surviving patients in the progressive disease group. Figure 2 of this report suggests that hepatic artery infusion may have had an influence on outcome. The time periods for both of these studies essentially overlap, and therefore both groups should have been able to offer similar therapies to their patients. The only apparent difference was the use of postoperative hepatic artery infusion in a proportion of the patients in Dr. Gallagher's study and preoperative chemoembolization in a proportion of the patients in Dr. Adam's study.

So what can we learn from Dr. Gallagher's study? This is the most comprehensive study to date specifically focused on synchronous disease. It suggests that, in this subset of patients with potentially resectable liver metastases, response to preoperative therapy is not predictive of outcome. Ultimately, a prospective, multi-institutional clinical registry is needed to confirm the results of this retrospective single-institution study. While a clinical trial may address the potential benefits of different approaches to therapy, it is hard to conceive of a trial that would specifically address the question posed in this study other than in a subgroup analysis. To date, only one randomized phase III trial of perioperative therapy versus surgery alone has been reported. ${ }^{5}$ A little over one-third of the patients included in this trial had synchronous disease, but a separate subgroup analysis of this group was not performed.

It therefore appears that, for the present time, we should offer surgery to all patients with potentially resectable disease, irrespective of their response to preoperative chemotherapy. We should instead try to learn what additional factor(s) influenced the positive outcome in the group of patients with progressive disease and allowed these patients the chance to have an overall survival similar to that achieved by patients with responsive disease. As noted by Dr. Adam in a recent review, "the management of synchronous liver metastases is changing." As we learn more from our past experiences we must reshape our practices to offer the best possible therapies to our patients with the right timing and sequence. That timing and sequence is yet to be fully defined. Given the difficulties of performing these studies it is imperative that multi-institutional studies and prospective registries are undertaken.

\section{REFERENCES}

1. Nordlinger B, Van Cutsem E, Rougier P, et al. Does chemotherapy prior to liver resection increase the potential for cure in patients with metastatic colorectal cancer? A report from the European Colorectal Metastases Treatment Group. Eur $J$ Cancer. 2007;43(14):2037-45.

2. Allen PJ, Kemeny N, Jarnagin W, et al. Importance of response to neoadjuvant chemotherapy in patients undergoing resection of synchronous colorectal liver metastases. J Gastrointest Surg. 2003;7(1):109-15; discussion 116-7.

3. Gallagher DJ, Zheng J, Capanu M, et al. Response to neoadjuvant chemotherapy does not predict overall survival for patients with synchronous colorectal hepatic metastases. Ann Surg Oncol. 2009.

4. Adam R, Pascal G, Castaing D, et al. Tumor progression while on chemotherapy: a contraindication to liver resection for multiple colorectal metastases? Ann Surg. 2004;240(6):1052-61; discussion 1061-4.

5. Nordlinger B, Sorbye H, Glimelius B, et al. Perioperative chemotherapy with FOLFOX4 and surgery versus surgery alone for resectable liver metastases from colorectal cancer (EORTC Intergroup trial 40983): a randomised controlled trial. Lancet. 2008;371(9617):1007-16. 